www.nature.com/jhg

\title{
Integration-free and stable expression of FVIII using a human artificial chromosome
}

\author{
Hajime Kurosaki ${ }^{1,2}$, Masaharu Hiratsuka ${ }^{3}$, Natsuko Imaoka ${ }^{1,2}$, Yuichi Iida ${ }^{1,2}$, Narumi Uno ${ }^{1,2}$, \\ Yasuhiro Kazuki $^{1,2}$, Chie Ishihara ${ }^{1,2,4}$, Yuwna Yakura ${ }^{4}$, Jun Mimuro ${ }^{5}$, Youichi Sakata ${ }^{5}$, Hiroyuki Takeya ${ }^{4}$ \\ and Mitsuo Oshimura ${ }^{1,2}$
}

Human artificial chromosome (HAC) has several advantages as a gene therapy vector, including stable episomal maintenance that avoids insertional mutations and the ability to carry large gene inserts. To examine the copy number effect on the gene expression levels and its stability for a long-term culture for a future application in gene therapy, we constructed a HAC vector carrying the human factor VIII (FVIII) complementary DNA, FVIII-HAC in Chinese hamster ovary (CHO) cells. One and more copies of $F$ VIII gene on the HAC were expressed in the copy-number-dependent manner in the CHO cells. The HAC with 16 copies of FVIII, FVIII (16)-HAC, was transferred from CHO hybrids into a human immortalized mesenchymal stem cell using microcell-mediated chromosome transfer. The expression levels of HAC-derived FVIII transgene products were compared with transfected FVIII plasmids. The former showed expression levels consistent with those of the original clones, even after 50 population doublings, whereas the latter showed a remarkable decrease in expression despite unvarying DNA content, indicating that the gene on the HAC is resistant to gene silencing. These results suggest that the HAC-mediated therapeutic gene-expression system may be a powerful tool for stable expression of transgenes, and possibly for industrial production of gene products.

Journal of Human Genetics (2011) 56, 727-733; doi:10.1038/jhg.2011.88; published online 11 August 2011

Keywords: factor VIII; hemophilia A; human artificial chromosome; microcell-mediated chromosome transfer

\section{INTRODUCTION}

Hemophilia A, an X-linked hemorrhagic disorder caused by mutations in the gene that encodes FVIII, affects 1 in 5000 males. Clinically, severe hemophilia is characterized by spontaneous and prolonged hemorrhage that can result in disability. Currently, treatment consists of factor replacement by using pooled FVIII concentrate or recombinant product. Although these protein replacement approaches are effective in many patients with mild and moderate hemophilia, they are limited by several factors, including the short in vivo halflife, high cost and scarcity of purified FVIII. Thus, gene therapy for hemophilia A is an attractive alternative to the protein replacement therapy. ${ }^{1}$

Optimal gene delivery vectors support long-term stable maintenance in host cells without integration into the host chromosomes, and they support appropriate spatial and temporal expression of therapeutic genes at desired levels in specific target cells; in addition, they do not pose the risk of cellular transformation or stimulate the host immune system. The use of currently available viral vector systems may be complemented by the use of human artificial chromosomes (HAC) because HAC replicates and segregates independently from host chromosomes as a minichromosome. ${ }^{2-6} \mathrm{HAC}$ can be transferred into different cell lines by microcell-mediated chromosome transfer. A HAC vector derived from human chromosome 21 and devoid of all endogenous genes is currently available. In addition, because HAC persists as a minichromosome, many complications associated with current vectors, including silencing of the therapeutic gene or oncogenesis resulting from integration in host chromosomes, may be minimized or eliminated. Thus, HAC has been offered as an alternative vector in cell-mediated gene therapy, and therapeutic genes have been transferred into multiple stem cell types via HAC, for example, genomic human p53 into multipotent germline stem cell ${ }^{7}$ and genomic Duchenne muscular dystrophy into mouse embryonic stem cell $^{5}$ and human and mouse induced pluripotent stem cells. ${ }^{6}$ HACs support long-term correction of defective genes because expression from and transmission of these vectors are stable throughout many cell divisions in human cells. ${ }^{4-8}$ This long-term stability is a significant improvement over the stability associated with current gene delivery methods.

\footnotetext{
${ }^{1}$ Department of Biomedical Science, Institute of Regenerative Medicine and Biofunction, Graduate School of Medical Science, Tottori University, Yonago, Japan; ${ }^{2}$ Chromosome Engineering Research Center, Tottori University, Yonago, Japan; ${ }^{3}$ Division of Molecular and Cell Genetics, Department of Molecular and Cellular Biology, School of Life Sciences, Faculty of Medicine, Tottori University, Yonago, Japan; ${ }^{4}$ Division of Pathological Biochemistry, Department of Life Sciences, Faculty of Medicine, Tottori University, Yonago, Japan and ${ }^{5}$ Division of Cell and Molecular Medicine, Center for Molecular Medicine, Jichi Medical University, School of Medicine, Tochigi, Japan

Correspondence: Professor M Oshimura, Department of Biomedical Science, Institute of Regenerative Medicine and Biofunction, Graduate School of Medical Science, Tottori University or Chromosome Engineering Research Center, Tottori University, 86, Nishi-cho, Yonago 683-8503, Japan.

E-mail: oshimura@med.tottori-u.ac.jp
}

Received 23 May 2011; revised 22 June 2011; accepted 11 July 2011; published online 11 August 2011 
In this study, we constructed HACs with multiple copies of the FVIII gene, FVIII-HACs, and compared the mitotic stability and transgene expression levels by the HAC vectors with those by a conventional vector in human immortalized mesenchymal stem cell (hiMSC), in order to examine the dosage effects on the expression levels of FVIII after a long-term culture.

\section{MATERIALS AND METHODS}

\section{Cell culture}

Hypoxanthine phosphoribosyl transferase (HPRT)-deficient Chinese hamster ovary (CHO; JCRB0218) hybrids containing the 21HAC2 vector were constructed previously ${ }^{9}$ and maintained in Ham's F-12 nutrient mixture (Invitrogen, Carlsbad, CA, USA) with $10 \%$ fetal bovine serum and $8 \mu \mathrm{g} \mathrm{ml}^{-1}$ blasticidin S (BS; Funakoshi, Tokyo, Japan). A hiMSC ${ }^{10}$ was maintained in Dulbecco's modified Eagle's medium (Sigma-Aldrich Inc., St Louis, MO, USA) supplemented with $10 \%$ fetal bovine serum (JRH Biosciences, Lenexa, KS, USA) ${ }^{5}$

\section{Construction of the FVIII-HAC using $\mathrm{CHO}$ cells}

A single copy of the FVIII expression cassette was $9.1 \mathrm{~kb}$ and composed of the following DNA elements: the HS4 insulator sequences derived from pCJ5-4 (gifted by Dr G Felsenfeld), ${ }^{11,12}$ the cytomegalovirus immediate early enhancer-chicken $\beta$-actin hybrid promoter ${ }^{13}$ (gifted by Dr M Okabe), a human B-domain deleted factor VIII (FVIII) $)^{14}$ and a bovine growth hormone poly A signal. FVIII-expression cassettes (1 to 16 copies) were inserted into the modified pPAC4 vector (see Supplementary Figure 1). Each modified pPAC4 plasmid with the FVIII-expression cassettes, abbreviated as FVIII-PAC, had a $3^{\prime}$ HPRT-loxP site, allowing the FVIII cassettes to transfer readily into the 21HAC2 vector. ${ }^{9}$ The $21 \mathrm{HAC} 2$ was stably maintained in the $\mathrm{CHO}$ clone in selective medium. The CHO cells with a $21 \mathrm{HAC} 2$ were co-transfected with $10 \mu \mathrm{g}$ of the modified pPAC4 vectors carrying the FVIII multicopy cassettes and $1 \mu \mathrm{g}$ of the Cre-expression vector, pBS185 (Invitrogen) using Lipofectamine 2000 reagent (Invitrogen) according to the manufacturer's protocol. After $24 \mathrm{~h}$ of culture in basic growth medium, the cells were cultured in medium containing hypoxanthine-aminopterin-thymidine (Sigma-Aldrich) for selection.

\section{Fluorescence in situ hybridization (FISH) analyses}

FISH analyses were performed using spreads of fixed chromosome in either metaphase or interphase from each cell hybrid, a digoxigenin-labeled (Roche, Basel, Switzerland) human COT-1 DNA probe (Invitrogen) and a biotinlabeled FVIII complementary DNA (cDNA) probe. Chromosomal DNA was counterstained with 4',6-diamidino-2-phenylindole (Sigma-Aldrich). Images were captured using the NIS-Elements system (Nikon, Tokyo, Japan).

\section{Microcell-mediated chromosome transfer and transfection}

Microcell-mediated chromosome transfer was performed as described previously. ${ }^{5} \mathrm{CHO}$ cells containing the FVIII-HAC were used as donor microcell hybrids. Briefly, hiMSC cells were fused with microcells prepared from donor hybrid $\mathrm{CHO}$ cells that contained FVIII-HAC, and hiMSC cells containing a FVIII-HAC were selected with BS $\left(4 \mu \mathrm{g} \mathrm{ml}^{-1}\right)$. The transferred FVIII-HAC in each line was characterized by PCR, reverse transcription-PCR (RT-PCR) and FISH analyses. The hiMSC cells were transfected with a linearized PAC containing the FVIII 16 copies cassettes and the bsd gene using Lipofectamine 2000 reagent (Invitrogen) according to the manufacturer's protocol. After $24 \mathrm{~h}$ of culture in basic growth medium, the cells were selected with BS $\left(4 \mu \mathrm{g} \mathrm{ml}^{-1}\right)$.

\section{Genomic PCR analyses}

Genomic DNA was extracted from cell lines using a genomic DNA extraction kit with DNAse-free RNase (Gentra Systems, Minneapolis, MN, USA). Primer sequences are listed in Supplementary Table. Primers used for amplification of the FVIII transgene spanned the intron and did not amplify a product in the host genomic DNA.

\section{Reverse transcription-PCR}

Total RNA was extracted using the Isogen reagent (Nippon Gene, Tokyo, Japan). Prepared RNA was treated with RNase-free DNase I (WAKO Jun-yaku,
Osaka, Japan) and further purified using RNeasy columns (Qiagen, Hilden, Germany) according to the manufacturer's instructions. First-strand cDNA synthesis was carried out using an oligo- $(\mathrm{dT})_{20}$ primer and the Super Script III reverse transcriptase (Invitrogen) according to the manufacturer's instructions. Primer sequences are listed in Supplementary Table. These primers spanned the introns and did not amplify a product from genomic DNA (data not shown).

\section{Enzyme-linked immunosorbent assay}

Human FVIII antibodies (monoclonal anti-FVIII light chain and monoclonal anti-FVIII heavy chain) and plasma-derived human FVIII (phFVIII) were kindly gifted from the Chemo-Sero-Therapeutic Research Institute (Kaketsuken, Kumamoto, Japan). In all, 96-well microtiter plates (Costar, Cambridge, MA, USA) were coated with $10 \mu \mathrm{g} \mathrm{ml}^{-1}$ monoclonal antibodies to FVIII light chain. After blocking with $1 \%$ bovine serum albumin and $0.01 \% \mathrm{NaN}_{3}$ in Trisbuffer saline containing $0.05 \%$ Tween 20 , samples or phFVIII $\left(150 \mathrm{U} \mathrm{mg}^{-1}\right)$ as a standard in Tris-buffer saline containing $0.05 \%$ Tween 20 and $0.2 \%$ bovine serum albumin was added. A standard curve was constructed by serial dilution of phFVIII from 250 to $3.91 \mu \mathrm{g} \mathrm{ml}^{-1}$. After $2 \mathrm{~h}$ incubation at room temperature, biotinylated monoclonal antibodies to FVIII were then added and incubated for $2 \mathrm{~h}$ at room temperature. Biotinylated secondary antibodies to FVIII heavy chain were detected by streptavidin-biotinylated horseradish peroxidase complex. The absorbance was measured at $460 \mathrm{~nm}$ to quantify the antigen in the original samples.

\section{Clotting assay}

FVIII activity levels in the conditioned medium of cells or phFVIII (Kaketsuken) as a standard were measured as thrombin generation by activation of the coagulation cascade. Assay components, including human FVIII-deficient plasma (Cosmo Bio, Tokyo, Japan), phospholipid and silicic acid (Sanko Junyaku, Tokyo, Japan), $\mathrm{CaCl}_{2}$ and Tris- $\mathrm{HCl}$ were mixed and incubated for $10 \mathrm{~min}$ at $37^{\circ} \mathrm{C}$. The substrate Boc-Val-Pro-Arg-MCA (Peptide Institute, Osaka, Japan) was added, and the fluorescence was measured with a spectrofluorometer (PerSeptive Biosystems CytoFluor4000, Applied Biosystem, Foster City, CA, USA) at $360 \mathrm{~nm} / 460 \mathrm{~nm}$. A standard curve was obtained by plotting known activities of phFVIII (Kaketsuken).

\section{RESULTS}

\section{Reconstruction of HAC with multicopies of FVIII expression} cassettes

The 21HAC2 vector contains a $5^{\prime}$ HPRT-loxP cloning site into which circular DNA can be reproducibility inserted using the Cre-loxP system. We integrated each FVIII cassette into a $21 \mathrm{HAC} 2$ vector (FVIII-HAC). Each FVIII-PAC construct with one or more copies of the FVIII sequences (Supplementary Figure 1) was co-transfected with a Cre expression plasmid into $\mathrm{CHO}$ cells bearing a 21HAC2. The expressed Cre-recombinase reconstituted a functional HPRT gene, which confers hypoxanthine-aminopterin-thymidine resistance, on the recombinant FVIII (rFVIII)-HACs (Figure 1). In all, 144 hypoxanthine-aminopterin-thymidine-resistant colonies were expanded and screened by PCR analysis with the primers for the FVIII transgene and the reconstructed HPRT gene. Of the 144 hypoxanthine-aminopterin-thymidine-resistant clones, 98 carried an intact FVIII-HAC. Of the 98 clones, 85 expressed FVIII, as confirmed by RT-PCR and enzyme-linked immunosorbent assay analysis. We recovered averaging $17 \mathrm{CHO}$ clones for each of a single copy and the 4 different multicopy $(1,2,4,8$ and 16 copies of FVIII) on the HAC, abbreviated as FVIII (1)-HAC, FVIII (2)-HAC, FVIII (4)-HAC, FVIII (8)-HAC and FVIII (16)-HAC, respectively. For each of the five types of FVIII-HACs, seven clones with the highest levels of FVIII expression were selected for FISH analysis. FISH analysis confirmed that a FVIII cDNA probe hybridized to a HAC and that each FVIII-HAC was present as an independent minichromosome in 26 of the $35 \mathrm{CHO}$ clones. The FVIII transgenes were integrated into the HAC and a host chromosome in other nine clones. Finally, 10 clones (2 for each type of FVIII-HAC) 


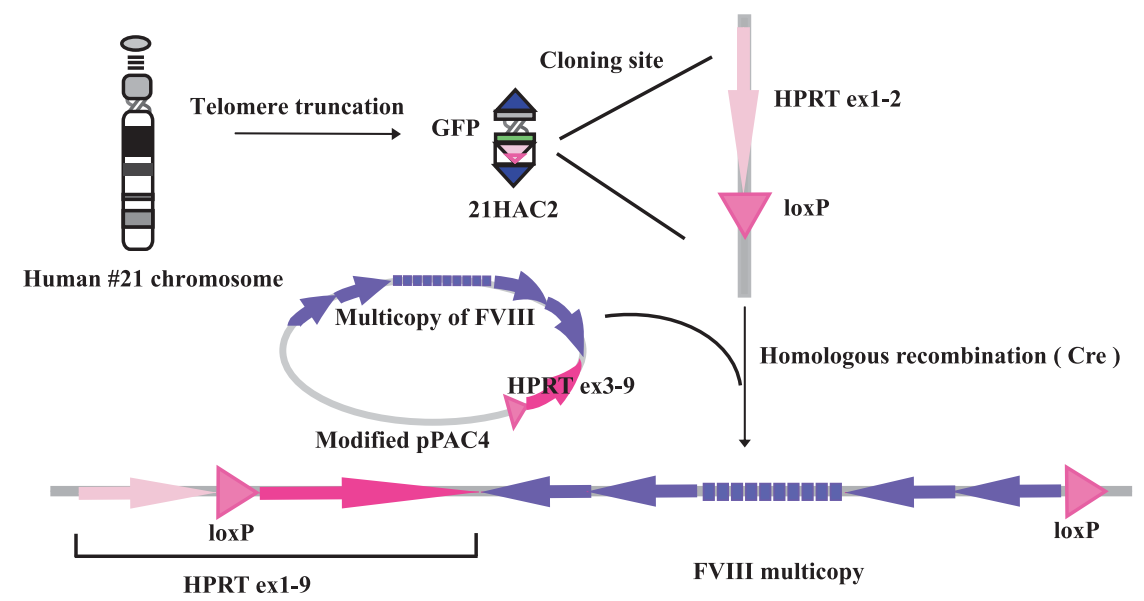

Figure 1 FVIII-HAC construction. The human chromosome 21-derived HAC vector (21HAC2) was previously established in HPRT-deficient Chinese hamster ovary clones. This HAC vector contains a cloning site, 5' HPRT-loxP, into which circular DNA can be reproducibility inserted with the Cre-loxP system. The modified pPAC4 with a FVIII-expression cassette (FVIII-PAC) has a 3' HPRT-IoxP site, thus allowing inserts from this PAC to transfer readily into the 21 HAC2 vector. The construction of FVIII-PAC is diagrammed in Supplementary Figure 1. The hypoxanthine-aminopterin-thymidine-resistant colonies should have the correct HAC insertions. 21HAC2, 21-derived human artificial chromosome 2 vector; FVIII, factor VIII; GFP, green fluorescent protein; HPRT, hypoxanthine phosphoribosyl transferase.

were randomly selected from the 26 clones. Only clones with a single correct insertion, as representative clones in Figure 2a, were used for further analyses. These results indicate that several FVIIIHACs with differing and defined numbers of FVIII genes were successfully constructed by cloning a defined FVIII cassette into the 21HAC2 vector.

\section{Expression levels of FVIII transgene}

The 10 clones selected for further analysis were subjected to PCR using the two primer sets; one set spanned the FVIII transgene, and the other spanned the site reconstructed within HPRT (Figure 2b). Genomic PCR analysis of FVIII sequences demonstrated that the copy number of the FVIII transgene in the HAC correlated with those in the PAC, which was transfected in CHO cells. Considering the FISH results in the 10 clones carrying a single and independent FVIII-HAC, the PCR analysis further confirmed that each FVIII-PAC construct had been correctly incorporated into a 21HAC2 to form different FVIIIHACs each with a specified number of FVIII genes.

Next, we examined whether FVIII expression levels correlated with FVIII copy number on the HAC in CHO clones. Expression of FVIII proteins was analyzed by enzyme-linked immunosorbent assay (Figure 2c). Each of the $10 \mathrm{CHO}$ clones carrying a FVIII-HAC were individually cultured until a confluent monolayer formed, and the amount of secreted FVIII present in the conditioned media was determined. The levels of secreted FVIII in the conditioned media correlated with the FVIII copy number in a dose-dependent manner in each $\mathrm{CHO}$ clone.

\section{Stable FVIII expression from FVIII-HACs in human cells}

The 21HAC2 vector is very stable in human cells, and mesenchymal stem cells hold promise for use in adult stem cell-mediated gene therapy. ${ }^{5}$ To investigate the transgene regulation and the mitotic stability of FVIII-HACs in a human cell line, two FVIII-HACs, FVIII (4)-HAC and FVIII (16)-HAC, were transferred separately from $\mathrm{CHO}$ cells into hiMSCs (Figure 3a). The hiMSC hybrids were selected with blasticidin $S$ and were screened by PCR, RT-PCR and FISH analyses. The FVIII-HACs were stably maintained in hiMSC clones, and they expressed FVIII mRNA, as determined by RT-PCR
(Figure 3b), and FVIII expression levels were higher in hiMSC clones with 16 FVIII copies than those in the clones with four FVIII copies.

Clotting assays using the conditioned media from the hiMSC cultures were also performed (Figure 3c), and the conditioned media from clones with 16 copies of FVIII performed better in the clotting assays than did those from the clones with 4 copies of FVIII. To confirm the function of human FVIII from the HAC (but not to examine the varidation of $\mathrm{CHO}$ cells for using cell therapy), CHO cells with the FVIII-HAC were intraperitoneally injected to the hemophilia model mice, ${ }^{15}$ and a tail clipping was performed whether model mice were rescued bleeding to death or not (Supplementary Figure 2 and Supplementary Data). These in vivo experiments showed that the FVIII-HAC contributed to expression of functional FVIII.

To evaluate the stability of the FVIII transgene on and FVIII gene expression from these FVIII (16)-HAC, the hiMSC cells were transfected with a PAC with 16 copies of the FVIII transgene and a BS-resistant gene (Figure 4a). Then, we selected stable transfectants. Single BS-resistant clones were expanded and subjected to PCR and RT-PCR analyses to assess the structure of the FVIII transgenes and the FVIII mRNA expression level, respectively, in each BS-resistant clone. Of 53 BS-resistant clones, four clones with a higher FVIII copy number were identified by PCR. The FVIII transgene mRNA expression levels were also higher in these four clones than in the others. Each independent hiMSCs line with a FVIII-HAC or a FVIII-PAC was cultured in medium with BS and separately in medium lacking BS. FISH analysis was performed after 50 population doublings (PDLs) of selected and unselected cultures to assess the mitotic stability of the FVIII-HACs. Individual intact FVIII-HACs were independently observed in metaphase cells after 50 PDLs in each culture, whereas metaphase figures from cultures derived from the FVIII-PAC transfectants showed that the FVIII-PAC had integrated into host chromosomes (Figure 4b). PCR analyses revealed that both FVIII-HAC sequences and FVIII-PAC sequences were retained under the unselected condition for the hiMSC cultures (Figures $4 \mathrm{c}$ and d). The FVIII mRNA expression levels were stable in clones with FVIII-HAC from 2 to 50 PDLs. Meanwhile, FVIII mRNA expression levels declined over the course of 50 PDLs in the hiMSC cultures derived from FVIII-PAC 

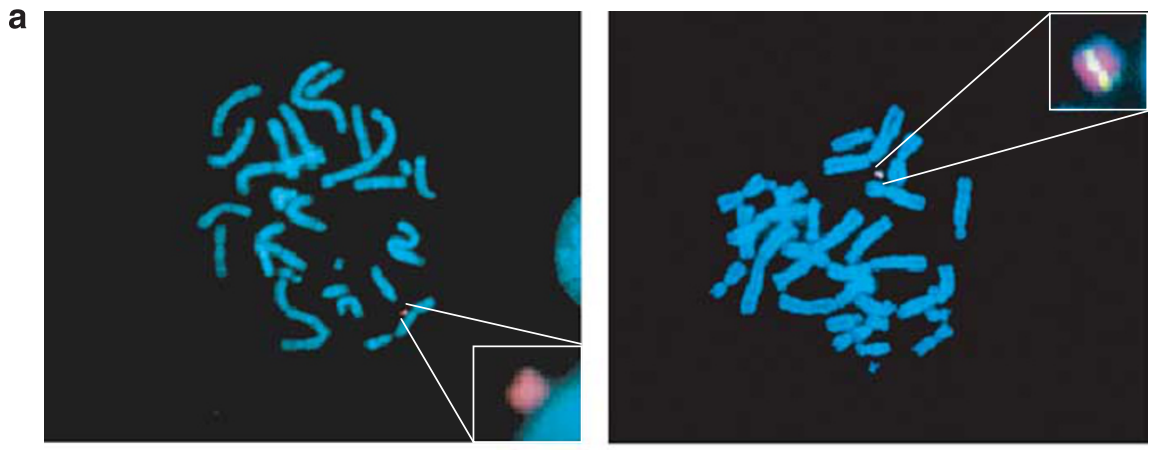

b

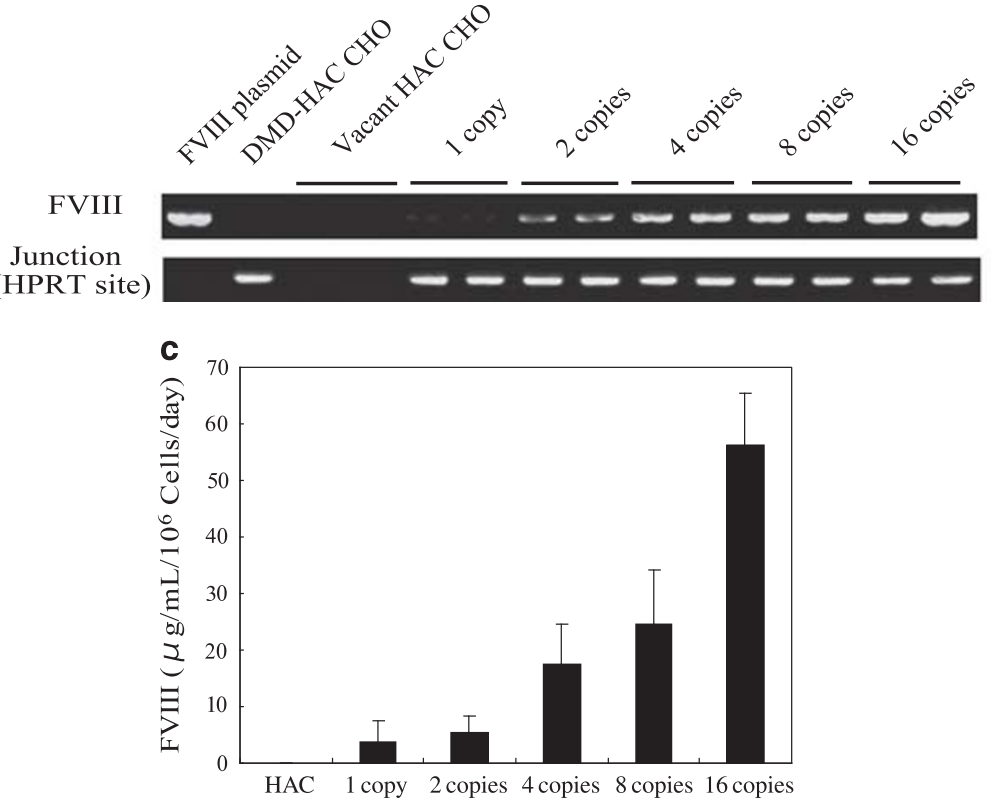

Figure 2 Reconstruction of multicopy FVIII on HAC in $\mathrm{CHO}$ cells. (a) Representative data from fluorescence in situ hybridization analysis of $\mathrm{CHO}$ cells carrying the 21 HAC2 vector (left panel) and the FVIII (8)-HAC (right panel). Human chromosome 21-derived alphoid DNA probe (red color) was hybridized to the HAC vector (right and left panels). FVIII probe (green signal) was hybridized to HAC vector in the CHO clone carrying the FVIII (8)-HAC (right panel). Chromosomal DNA was counterstained with 4',6-diamidino-2-phenylindole (blue). The inset shows an enlargement of $21 \mathrm{HAC2}$ with and without an FVIII insert. Images are representative of the results from the other $\mathrm{CHO}$ clones containing $21 \mathrm{HAC} 2$ and FVIII (1, 2, 4, 8 or 16)-HACs. (b) Representative data from PCR analysis of genomic DNA confirm the structure of the reconstituted HPRT junction and the FVIII transgenes. The FVIII plasmid was used as a positive control for the FVIII transgene (upper panel). The vacant 21HAC2 $\mathrm{CHO}$ (CHO 6TGr) clones were used as a negative control for the FVIII transgene and HPRT. DMD-HAC CHO clones $^{5}$ were used as a positive control for HPRT (lower panel). (c) Enzyme-linked immunosorbent assay was used to determine the FVIII protein concentration. FVIII was secreted into conditioned media (CM) from CHO clones with FVIII-HAC. Each clone was cultured in 12-well culture plates to full confluence in triplicate under the same conditions. The concentration of human FVIII antigen in CM was quantified by enzyme-linked immunosorbent assay, as described in Materials and Methods. HAC-selected $\mathrm{CHO}$ clones with a vacant $21 \mathrm{HAC} 2$ were used as a negative control. The bar graph represents the average amount of FVIII secreted from $\mathrm{CHO}$ clones with the indicated FVIII-HAC. CHO, Chinese hamster ovary clone; DMD, Duchenne muscular dystrophy; FVIII, factor VIII; HAC, human artificial chromosome; HPRT, hypoxanthine phosphoribosyl transferase.

transfectants with or without selection. These results indicate that the FVIII-HACs were mitotically stable and they did not integrate into the host chromosomes; moreover, FVIII transgene expression from FVIII-HACs persisted in the human cells even after long-term culture in vitro.

\section{DISCUSSION}

We developed HACs with multiple copies of a human FVIII transgene that may be useful for the gene expression. We assessed several characteristic of these HACs, which each carried one or more copies of the FVIII transgene, as a step toward producing recipient cells with controllable expression for use in cell-based transgene therapies. Our results showed that FVIII expression levels in CHO cells depended on the FVIII copy number on the HAC; specifically, $\mathrm{CHO}$ cells with a FVIII (16)-HAC expressed more protein than four types of CHO cells with FVIII (1, 2, 4 or 8)-HACs. Similarly, we showed that hiMSC hybrids bearing FVIII (16)-HAC supported higher transgene expression than those bearing FVIII (4)-HAC. The HAC with more copies of the FVIII transgene apparently support higher levels of FVIII expression.

A non-viral transgene delivery method consisting of transplantation of FVIII-transduced autologous fibroblasts was tested in a clinical trial. ${ }^{16}$ The data acquired from this phase I clinical trial showed only a modest and temporary indication of positive effects, suggesting that there is a relationship between durable transgene expression and transgene silencing. Moreover, the site of integration of a transgene into a host chromosome has a major effect on the transgene transcription rate, a phenomenon called a position effect. ${ }^{17}$ 
a

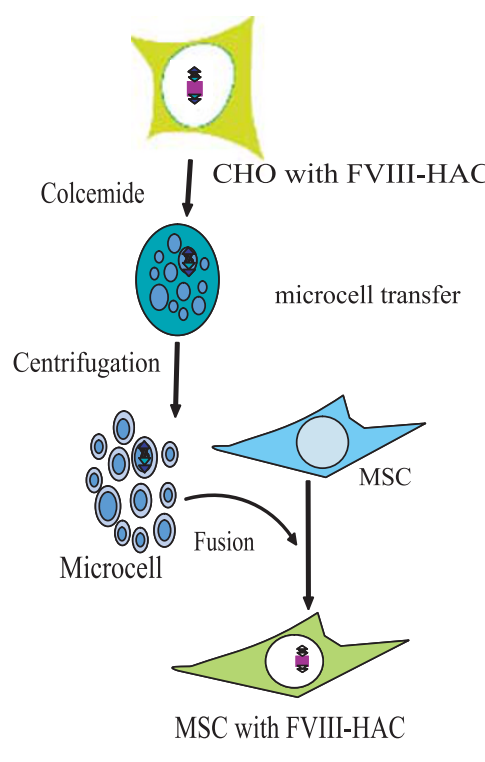

b

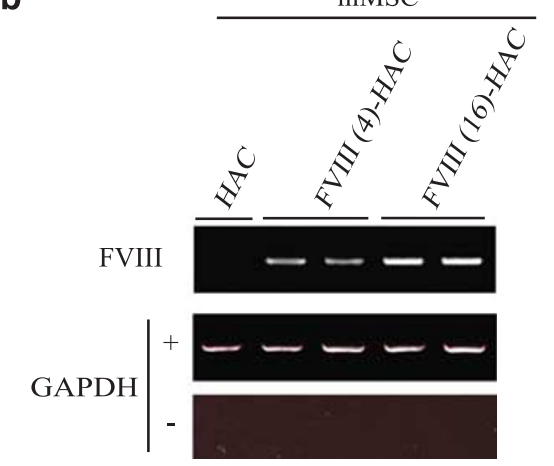

C

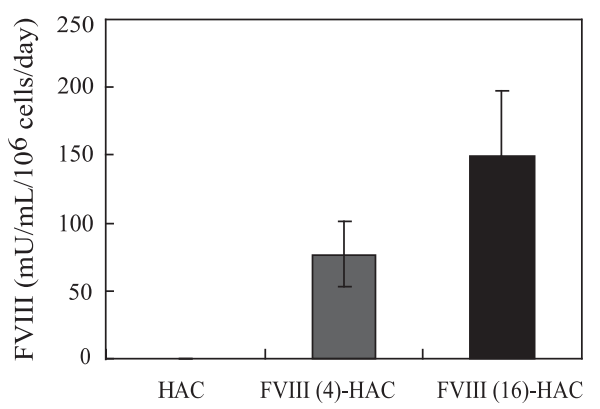

Figure 3 Analysis of the FVIII-HAC after transfer into hiMSC. (a) Schematic representation of the principle of microcell-mediated chromosome transfermediated FVIII-HAC transfer to hiMSC. Vacant 21HAC2, FVIII (4)-HAC or FVIII (16)-HAC from each of two CHO clones were transferred into hiMSCs by microcell-mediated chromosome transfer. Briefly, this method consists of four essential steps: micronucleation of the donor hybrids by colcemid treatment, enucleation in the presence of cytochalasin B, purification of the microcells and fusion with $1 \times 10^{6}$ recipient hiMSC cells. hiMSC hybrids were selected in $4 \mu \mathrm{gl}^{-1}$ blasticidin S and picked for expansion. Blasticidin S-resistant clones were screened by PCR analysis for the FVIII transgene and the HPRT junction and by reverse transcription-PCR analysis for FVIII expression. In all, 9 of the 14 hybrids with a FVIII (4)-HAC and 5 of 6 hybrids with a FVIII (16)-HAC were recovered. Four of the four hybrids with vacant HAC donors were recovered as blasticidin S-resistant and green florescent protein-positive clones. No rearrangements were observed in metaphase figures in any of these clones based on the fluorescence in situ hybridization analysis. (b) Analysis of FVIII and GAPDH mRNA by reverse transcription-PCR. Total RNA was prepared from CHO clones maintaining a vacant 21 HAC2 (1 clone), a FVIII (4)-HAC (2 clones) and a FVIII (16)-HAC (2 clones) PCR products obtained by using the primers for the FVIII transgene and GAPDH are shown. In the control reverse transcription-PCR reactions, a primer pair for GAPDH was added, but the complementary DNA template was not, and reverse transcription-PCR products were not amplified even after 35 cycles. (c) FVIII activity on the hiMSC clones by clotting assay. FVIII expression in CM of hiMSC clones with FVIII-HAC. The four $21 \mathrm{HAC} 2$ hybrids, nine FVIII (4)-HAC hybrids and five FVIII (16)-HAC hybrids were cultured in $6 \mathrm{~cm}$ dish culture plates to full confluence. The culture medium was replaced, and after $24 \mathrm{~h}$, the CM was harvested. FVIII activity levels in CM were measured as thrombin generation, as described in Materials and Methods. hiMSC clones maintaining $21 \mathrm{HAC} 2$ were used as the negative controls for the FVIII activity. The bar graph represents the average FVIII activity level of each FVIII-HAC clones with the indicated FVIII copy number. CHO, Chinese hamster ovary clone; GAPDH, glyceraldehyde-3-phosphate dehydrogenase; FVIII, factor VIII; HAC, human artificial chromosome; hiMSC, human immortalized mesenchymal stem cell; MSC, mesenchymal stem cell.

Numerous studies using viral vectors or cDNA plasmids to generate transgene-expressing clones have observed repression of transgene expression. ${ }^{18-22}$ In this study, $\geqslant 92 \%$ FVIII-PACs randomly integrated into the hiMSC host genome, whereas independently segregating FVIII-HACs persisted in $\geqslant 90 \%$ of the cells as determined by FISH analysis. Therefore, our results suggested that reductions in transgene expression in the FVIII-PAC transfected hiMSC cells were caused by silencing rather than by loss of the transgene itself. Furthermore, our results demonstrated that hiMSC could stably maintain HACs carrying multiple copies of a FVIII transgene and that this transgene was resistant to silencing when present on a HAC. HAC vectors may support persistent transgene expression, thereby minimizing the need for cell reimplantation because of a stable expression system that depends on a less invasive delivery method and is not influenced by position effects associated with integration sites.

rFVIII is one of the most complex mammalian proteins, and a biotechnology venture may be important to the development of a treatment for hemophilia A. HAC vectors may support persistent transgene expression from our results, thereby these donor $\mathrm{CHO}$ clones may also be useful for biopharmaceutical production of rFVIII. The stability of cell lines in the absence of selection pressure is important to ensure predictable and high yields of recombinant protein during production. ${ }^{23}$ We examined FVIII production in cultures of CHO cells carrying FVIII-HACs in the absence of selec tion and over the long term. Some $\mathrm{CHO}$ clones gradually lost the FVIII-HAC; nevertheless, hiMSC hybrid clones retained the same FVIII-HAC over the long term. The current study did not address the mechanism by which host cell types, rodent or human, influence the retention rate for HAC maintenance. However, empirically, the $21 \mathrm{HAC} 2$ vector is very stable in human cells, ${ }^{5}$ in clear contrast to its instability in rodent cells. To establish an rFVIII production system in the future, the human hepatocellular carcinoma cell line (HepG2) or human immortalized hepatocytes could be used as the host cell because it is of hepatic origin, which may contribute to proper rFVIII processing and secretion. ${ }^{24-25}$ 


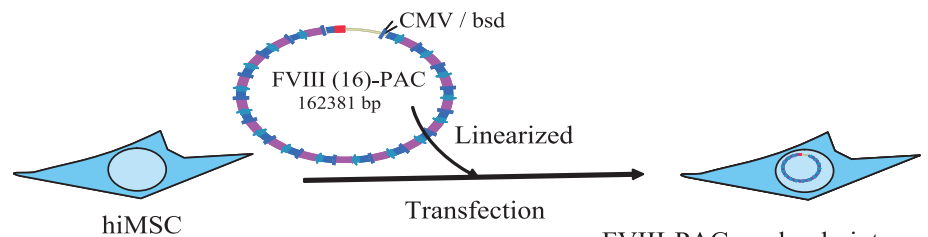

FVIII-PAC randomly integrated clone

b

FVIII (16)-HAC transfctant FVIII (16)-PAC transfectant
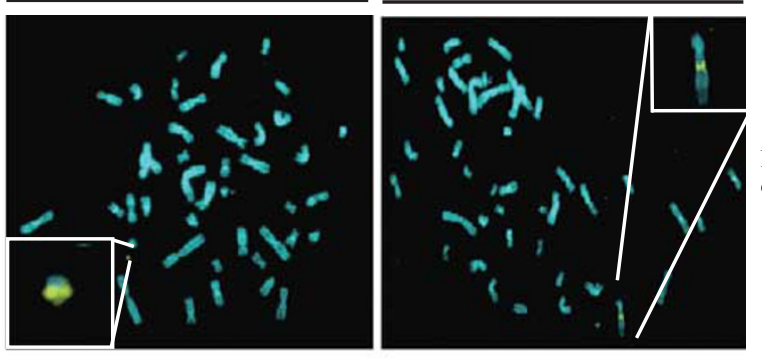

Blue : DAPI

Green : FVIII

C
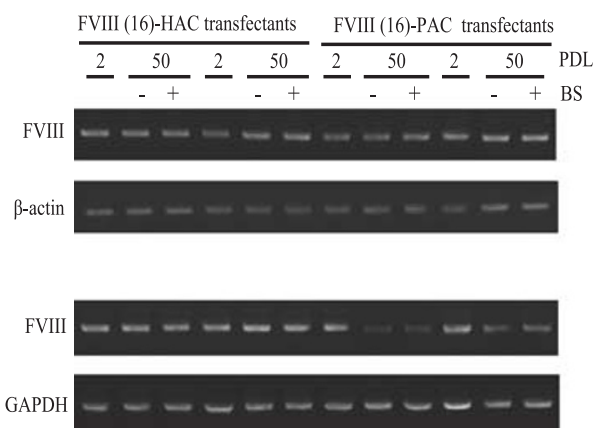

d
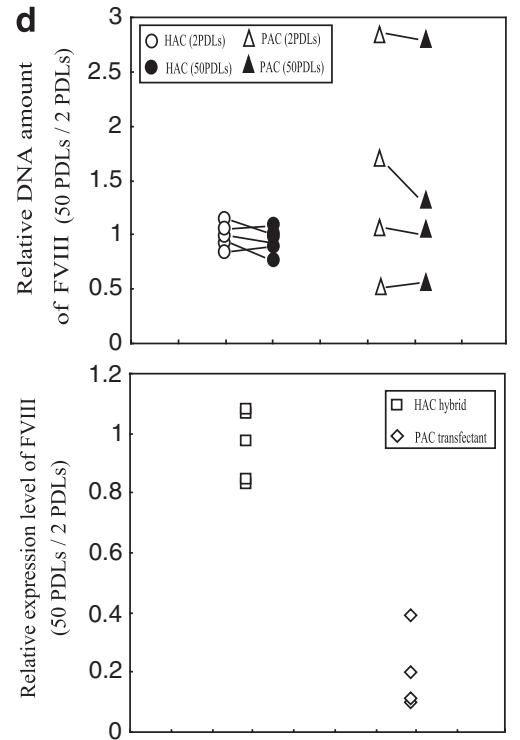

Figure 4 Transgene stability and sustained transgene regulation associated with FVIII-HAC and FVIII-PAC in hiMSC. (a) Flow diagram of transfection with FVIII-PAC containing a blasticidin S-resistance gene into hiMSC. hiMSC cells were transfected with a FVIII (16)-PAC containing a blasticidin S-resistance gene. Single clones were expanded, examined for the FVIII transgene by PCR and tested for FVIII mRNA expression levels by reverse transcription-PCR. The top 4 clones among 53 clones were selected because the FVIII transgene was more abundant. The gene expression levels of FVIII in these 4 clones were higher than those in the other 49 clones. (b) Typical metaphase fluorescence in situ hybridization images of FVIII-HAC clones and FVIII-PAC clones. Fluorescence in situ hybridization analyses were performed using either fixed metaphase or interphase spreads of FVIII-HAC clones and FVIII-PAC clones and biotin-labeled FVIII complementary DNA. Chromosomal DNA was counterstained with DAPI (Sigma-Aldrich). Images were captured using the NIS-Elements system (Nikon). Enlarged areas indicate a FVIII-HAC (left panel) and a FVIII-PAC that had integrated into the host genome (right panel). The endogenous chromosomes in hiMSC clones with FVIII-HAC had not undergone any rearrangement after 50 PDLs based on the metaphase spreads; in contrast, the FVIII transgene had integrated into endogenous host chromosomes in the FVIII-PAC clones. (c) Transgene content and expression levels in FVIII-HAC clones and FVIII-PAC transfectants. hiMSC hybrids with a FVIII (16)-HAC or with randomly integrated FVIII (16)-PAC clones were maintained with $(+)$ or without (-) $4 \mu \mathrm{g} \mathrm{ml}^{-1}$ blasticidin S for 50 PDLs. Relative quantification of the FVIII transgene and $\beta$-actin (human $\beta$ actin) on genomic PCR (upper side). After the initial stages of cloning and long-term culture, total genomic DNA was prepared from FVIII (16)-HAC transferred into hiMSC clones and FVIII (16)-PAC transfectants. Analysis of FVIII and GAPDH mRNAs by reverse transcription-PCR is shown in the lower panel. After the initial stages of cloning and long-term culture, total RNA was prepared from hiMSC clones with a FVIII (16)-HAC and from FVIII (16)-PAC transfectants. (d) Graphic representation of the FVIII transgene and its expression in hiMSC hybrids and transfectants after 2 (initial stage of cloning) and 50 PDLs (long-term culture after selection withdrawal) based on the results of (c). Genomic PCR analysis of FVIII and the $\beta$-actin control is shown in the upper graph. The number of FVIII sequences in one of the five hiMSC hybrids clones at 2PDLs was used as a standard. Reverse transcription-PCR analysis of relative FVIII expression at 50 PDLs to 2 PDLs is shown in the lower graph. FVIII expression levels at 50 and 2 PDLs were standardized using GAPDH expression, and the relative FVIII expression in each clone at 2PDLs was used as the control. CMV, cytomegalovirus; DAPI, 4',6-diamidino-2-phenylindole; GAPDH, glyceraldehyde3-phosphate dehydrogenase; FVIII, factor VIII; HAC, human artificial chromosome; hiMSC, human immortalized mesenchymal stem cell; PDLs, population doublings. 


\section{ACKNOWLEDGEMENTS}

We wish to thank Drs M Okabe (Osaka University) for providing pCX-EGFP; G Felsenfeld, (National Institutes of Health) for providing pJC5-4. This study was supported in part by a Health and Labor Science Research Grant for Research on HIV/AIDS and Research on Intractable Diseases from the Japanese Ministry of Health, Labour and Welfare, a Grant-in-Aid for Research Activity Start-up, and a Regional Innovation Cluster Program grant (accelerative support) from the Ministry of Education, Culture, Sports, Science and Technology of Japan. All animal studies were approved by the Institutional Animal Care and Use Committee of Tottori University.

1 Murphy, S. L. \& High, K. A. Gene therapy for haemophilia. Br. J. Haematol. 140, 479-487 (2008).

2 Basu, J. \& Willard, H. F. Artificial and engineered chromosomes: non-integrating vectors for gene therapy. Trends Mol. Med. 11, 251-258 (2005).

3 Oshimura, M. \& Katoh, M. Transfer of human artificial chromosome vectors into stem cells. Reprod. Biomed. Online 16, 57-69 (2008).

4 Katoh, M., Ayabe, F., Norikane, S., Okada, T., Masumoto, H., Horike, S. et al. Construction of a novel human artificial chromosome vector for gene delivery. Biochem. Biophys. Res. Commun. 321, 280-290 (2004).

5 Hoshiya, H., Kazuki, Y., Abe, S., Takiguchi, M., Kajitani, N., Watanabe, Y. et al. A highly stable and nonintegrated human artificial chromosome (HAC) containing the $2.4 \mathrm{Mb}$ entire human dystrophin gene. Mole. Ther. 17, 309-317 (2009).

6 Kazuki, Y., Hiratsuka, M., Takiguchi, M., Osaki, M., Kajitani, N., Hoshiya, H. et al. Complete genetic correction of iPS cells from duchenne muscular dystrophy. Mole. Ther. 18, 386-393 (2010).

7 Kazuki, Y., Hoshiya, H., Kai, Y., Abe, S., Takiguchi, M., Osaki, M. et al. Correction of a genetic defect in multipotent germline stem cells using a human artificial chromosome. Gene Ther. 15, 617-624 (2008).

8 Ren, X. Y., Tahimic, C. G. T., Katoh, M., Kurimasa, A., Inoue, T. \& Oshimura, M. Human artificial chromosome vectors meet stem cells - new prospects for gene delivery. Stem Cell Rev. 2, 43-50 (2006).

9 Kazuki, Y., Hoshiya, H., Takiguchi, M., Abe, S., lida, Y., Osaki, M. et al. Refined human artificial chromosome vectors for gene therapy and animal transgenesis. Gene Ther. 18, 384-393 (2011).

10 Okamoto, T., Aoyama, T., Nakayama, T., Nakamata, T., Hosaka, T., Nishijo, K. et al. Clonal heterogeneity in differentiation potential of immortalized human mesenchymal stem cells. Biochem. Biophys. Res. Commun. 295, 354-361 (2002).

11 Chung, J. H., Bell, A. C. \& Felsenfeld, G. Characterization of the chicken beta-globin insulator. Proc. Natl Acad. Sci. U S A 94, 575-580 (1997).

12 Recillas-Targa, F., Pikaart, M J., Burgess-Beusse, B., Bell, A C., Litt, M D., West, A G. et al. Position-effect protection and enhancer blocking by the chicken beta-globin insulator are separable activities. Proc. Natl Acad. Sci. U S A 99, 6883-6888 (2002).

13 Niwa, H., Yamamura, K. \& Miyazaki, J. Efficient selection for high-expression transfectants with a novel eukaryotic vector. Gene 108, 193-199 (1991).

14 Ogata, K., Mimuro, J., Kikuchi, J., Tabata, T., Ueda, Y., Naito, M. et al. Expression of human coagulation factor VIII in adipocytes transduced with the simian immunodeficiency virus agmTYO1-based vector for hemophilia A gene therapy. Gene Ther. 11, 253-259 (2004).

15 Bi, L., Lawler, A. M., Antonarakis, S. E., High, K. A., Gearhart, J. D. \& Kazazian, H. H. Targeted disruption of the mouse factor-viii gene produces a model of hemophilia-A. Nat. Genet. 10, 119-121 (1995).

16 Roth, D. A., Tawa, N. E., O'Brien, J. M., Treco, D. A., Selden, R. F. \& Factor, V. T. T. Nonviral transfer of the gene encoding coagulation factor VIII in patients with severe hemophilia A. N. Engl. J. Med. 344, 1735-1742 (2001).

17 Wilson, C., Bellen, H. J. \& Gehring, W. J. Position effects on eukaryotic geneexpression. Ann. Rev. Cell Biol. 6, 679-714 (1990).

18 Ellis, J. Silencing and variegation of gammaretrovirus and lentivirus vectors. Hum. Gene Ther. 16, 1241-1246 (2005).

19 Hong, H., Takahashi, K., Ichisaka, T., Aoi, T., Kanagawa, O., Nakagawa, M. et al. Suppression of induced pluripotent stem cell generation by the p53-p21 pathway. Nature 460, 1132-U1195 (2009).

20 Takahashi, K. \& Yamanaka, S. Induction of pluripotent stem cells from mouse embryonic and adult fibroblast cultures by defined factors. Cell 126, 663-676 (2006).

21 Okita, K., Nakagawa, M., Hong, H. J., Ichisaka, T. \& Yamanaka, S. Generation of mouse induced pluripotent stem cells without viral vectors. Science 322, 949-953 (2008).

22 Takahashi, K., Tanabe, K., Ohnuki, M., Narita, M., Ichisaka, T., Tomoda, K. et al. Induction of pluripotent stem cells from adult human fibroblasts by defined factors. Cell 131, 861-872 (2007).

23 Wurm, F M. Production of recombinant protein therapeutics in cultivated mammalian cells. Nat. Biotechnol. 22, 1393-1398 (2004).

24 Herlitschka, S. E., Schlokat, U., Falkner, F. G. \& Dorner, F. High expression of a B-domain deleted factor VIII gene in a human hepatic cell line. J. Biotechnol. 61, 165-173 (1998).

25 Campos-da-Paz, M., Costa, C. S., Quilici, L. S., de Carmo Simões, I., Kyaw, C. M., Maranhão, A. Q. et al. Production of recombinant human factor VIII in different cell lines and the effect of human XBP1 co-expression. Mol. Biotechnol. 39, 155-158 (2008).

Supplementary Information accompanies the paper on Journal of Human Genetics website (http://www.nature.com/jhg) 